\title{
Editorial: Professional Training in Extended Reality: Challenges and Solutions
}

\author{
Lap-Fai Yu ${ }^{1 *}$, Christos Mousas ${ }^{2}$, David Lindlbauer ${ }^{3}$, George Alex Koulieris ${ }^{4}$, Liwei Chan ${ }^{5}$, \\ Ryan P. McMahan ${ }^{6}$ and Tomohiro Amemiya ${ }^{7}$ \\ ${ }^{1}$ George Mason University, Fairfax, VA, United States, ${ }^{2}$ Purdue University, West Lafayette, IN, United States, ${ }^{3}$ Carnegie Mellon \\ University, Pittsburgh, PA, United States, ${ }^{4}$ Durham University, Durham, United Kingdom, ${ }^{5}$ National Yang Ming Chiao Tung \\ University, Hsinchu, Taiwan, ${ }^{6}$ University of Central Florida, Orlando, FL, United States, ${ }^{7}$ The University of Tokyo, Tokyo, Japan
}

Keywords: virtual reality, training, augmented reality, virtual environments, content creation

\section{Editorial on the Research Topic}

Professional Training in Extended Reality: Challenges and Solutions

Professional training is the most common usage of extended reality (XR) technologies in enterprises. For example, Walmart has used virtual reality to train more than one million employees. The immersiveness, interactivity, and realism offered by XR-based professional training make it more effective and engaging than traditional flat training media such as instruction manuals and videos, leading to a reduction in training time and a significant improvement in performance. The convenience, safety, scalability, controllability, and low-cost nature of XR-based professional training also make it highly promising as an approach for public service training, fitness training, home-schooling, and rehabilitation.

While XR-based professional training is promising, a number of technical challenges need to be solved to facilitate its widespread adoption and effective use. Through this research topic, we aimed to articulate visions on overcoming the research challenges of using extended reality technologies for professional training purposes. We accepted research papers in a variety of computer disciplines related to XR-based professional training and content creation to inspire the field to further explore this promising direction.

\section{CHALLENGES AND SOLUTIONS OF XR FOR PROFESSIONAL TRAINING}

Franzluebbers et al. proposed a virtual reality simulator for veterinary neurology training. Through a user study, the authors evaluated interaction styles for such novel training application by comparing a reality-based interaction metaphor with a command-based metaphor. Based on their findings the authors observed a preference of participants for the reality-based system. They also found that the inclusion of haptic feedback could improve usability issues.

Pedram et al. conducted two successive rounds of virtual mines rescue training; the first in a surround projection environment (360-VR) and the second in desktop virtual reality (Desktop-VR). The results of the conducted study suggested that the Learning Context (non-immersive VR) was the most important factor that contributed to the positive learning outcome of their participants as well as that the participants of this study preferred the Desktop-VR as a training environment. In the paper, the authors also discuss that well-suited technologies to the training task could be more appropriate compared to "more advanced" ones (e.g., Desktop-VR could be considered more 
suitable compared to $360-\mathrm{VR}$ when users act individually). Finally, the authors argue that training-related factors such as the quality of human trainers are critical for virtual reality training.

Xie et al. review virtual reality skill training applications. First, the authors describe the different ways that virtual reality training experiences are created and delivered using various software and hardware solutions. Second, the authors discuss the challenges and solutions of virtual reality training in first responders, medical, military, workforce, and education domains. Then, they discuss different methods that can be used to validate the effectiveness of virtual reality training and conclude by discussing future potential directions for developing novel virtual reality training experiences.

Pavlou et al. developed an XR platform for creating and delivering training programs targeting industrial settings. The proposed platform is composed of various modules including the creation and execution of XR tutorials, the simulation module using a digital human model, and the biophysics assessment. Moreover, a drilling tutorial execution study was also conducted and showed a satisfactory perception of participants on the XR training process.

Engelbrecht and Hoermann present a systematic review that summarizes the existing literature on virtual reality firefighting training mainly focusing on human factors and learning outcomes. The outcome of this systematic review indicates the importance and suitability of virtual reality on firefighter training since it is shown that such training environments can be considered analog to real-life training. Lastly, the authors discuss the need to establish clear evidence and guidelines on how virtual reality training could be optimized to increase reliable data through appropriate research endeavors.

Tavassoli et al. designed and developed a training platform with a pediatric virtual patient to teach medical learners the spectrum of symptoms and severity levels of Autism Spectrum Disorder in young children. The authors also conducted a user study with pediatric trainees to evaluate the effectiveness of the platform. The results obtained from their study indicated that training with the presented platform improved the trainees' performance in careful observation and accurate classification of real children's behaviors in video vignettes. Lastly, the authors discuss the applicability of their platform to enhance professional training for early detection of autism in young children.
Li et al. conducted a study to explore the effect of different human factors, such as domain knowledge, culture, and familiarity of virtual reality technologies, on the perception of virtual operating room experience. By collecting subjective ratings and qualitative feedback from study participants, the authors found that the surgical knowledge only affected perceived mental demand when using a virtual operating room. Moreover, the authors also report their findings on cultural differences and the virtual reality experience of participants demonstrating the influences of cultural differences on the perception of immersion and usability.

\section{CONCLUSION}

Through this research topic, a number of papers were published which provide insights on using extended reality technologies for professional training. With the growing popularity and advancements of virtual reality and mixed reality devices, we believe that using extended reality for professional training will become a promising and common training solution in the years to come.

\section{AUTHOR CONTRIBUTIONS}

All authors listed have made a substantial, direct, and intellectual contribution to the work and approved it for publication.

Conflict of Interest: The authors declare that the research was conducted in the absence of any commercial or financial relationships that could be construed as a potential conflict of interest.

Publisher's Note: All claims expressed in this article are solely those of the authors and do not necessarily represent those of their affiliated organizations, or those of the publisher, the editors and the reviewers. Any product that may be evaluated in this article, or claim that may be made by its manufacturer, is not guaranteed or endorsed by the publisher.

Copyright (c) 2021 Yu, Mousas, Lindlbauer, Koulieris, Chan, McMahan and Amemiya. This is an open-access article distributed under the terms of the Creative Commons Attribution License (CC BY). The use, distribution or reproduction in other forums is permitted, provided the original author(s) and the copyright owner(s) are credited and that the original publication in this journal is cited, in accordance with accepted academic practice. No use, distribution or reproduction is permitted which does not comply with these terms. 